\title{
The Effect of Haloperidol Administration on Heart Rate Variability in Isolated Heart of Schizophrenia-like and Control Rats
}

\author{
Oto Janousek $^{1}$, Tibor Stracina ${ }^{2}$, Marina Ronzhina ${ }^{1}$, Jakub Hejc ${ }^{1}$, Tibor Stark ${ }^{3}$, Jana Ruda ${ }^{3}$, Vincenzo \\ Micale $^{4}$, Jana Kolarova ${ }^{1}$, Marie Novakova ${ }^{2}$, Ivo Provaznik ${ }^{1}$ \\ ${ }^{1}$ Department of Biomedical Engineering, Brno University of Technology, Brno, Czech Republic \\ ${ }^{2}$ Department of Physiology, Faculty of Medicine, Masaryk University, Brno, Czech Republic \\ ${ }^{3}$ Department of Pharmacology, Faculty of Medicine, Masaryk University, Brno, Czech Republic \\ ${ }^{4}$ Centre for Neuroscience, Central European Institute of Technology, Masaryk University, Brno, \\ Czech Republic
}

\begin{abstract}
Deeper understanding of heart rate variability $(H R V)$ changes induced by haloperidol administration can contribute to overcoming of adverse cardiovascular effects of antipsychotics. The effect of haloperidol administration on HRV was therefore studied in nine schizophrenia-like rat isolated hearts and six control rat hearts.

Isolated hearts were stabilized (20 min) on Langendorff perfusion system, then exposed to haloperidol $(40 \mathrm{~min})$ and finally washed out $(40 \mathrm{~min})$. The effect of haloperidol administration was studied in tachograms obtained from touchless-recorded electrograms (5 kHz, 12-bit).

$H R V$ parameters evolution in time, frequency, geometric, and non-linear domain were computed from 10 minutes long tachogram segments during stabilization, haloperidol exposition and washout periods.

The haloperidol administration significantly affected $H R V$ parameters in control rat hearts, but it had limited effect on schizophrenia-like ones. The effect of haloperidol administration was irreversible, since the washout did not restore HRV parameters to their initial values.
\end{abstract}

\section{Introduction}

Schizophrenia is a mental disease with increasing incidence worldwide. For the treatment of majority of schizophrenics, antipsychotics are drugs of the first choice. However, antipsychotic drugs may affect, among others, cardiovascular system performance resulting in health worsening.

Classic antipsychotic drug haloperidol is known to have a proarrhythmogenic effect $[1,2]$. However, the mechanism of this effect has not been clearly elucidated. Moreover, impairment of heart function in treated as well as drug-free schizophrenic patients was previously reported [3].
The aim of this study was to find out whether hearts of schizophrenia-like rats differ in HRV parameters from control rat hearts during administration of haloperidol and after its washout.

\section{Methods}

For schizophrenia modelling, neurodevelopmental methylazoxymethanol acetate rat model of schizophrenia was used [4]. Fifteen Sprague-Dawley adult rats (male only) were included in the study: six in control group $(\mathrm{CON})$ and nine in schizophrenia-like group $(\mathrm{SCH})$. Animals were housed in groups of two under controlled environmental conditions with food and water available $a d$ libitum. All experiments followed the guidelines for animal treatment approved by European Community Guide for the Care and Use of Laboratory Animals and the study was approved by the local authority Committee on the Protection of Animals, Faculty of Medicine, Masaryk University.

\subsection{Isolated hearts}

For isolated heart experiment, 32 weeks old rats with body mass $547 \pm 68 \mathrm{~g}$ were used. In deep anaesthesia, the heart was rapidly excised, placed in a cold $\left(4^{\circ} \mathrm{C}\right) \mathrm{Krebs}-$ Henseleit (K-H) solution and prepared for cannulation. The aorta was cannulated and the heart was perfused according to Langendorff at constant perfusion pressure $(75 \mathrm{mmHg}$ ) with $\mathrm{K}-\mathrm{H}$ solution $(\mathrm{NaCl}, 118 \mathrm{mM}$; $\mathrm{NaHCO} 3$, $27 \mathrm{mM}$; KCl, $4.8 \mathrm{mM}$; KH2PO4, $1.0 \mathrm{mM}$; MgSO4, 1.2 $\mathrm{mM}$; CaCl2, $1.2 \mathrm{mM}$; glucose, $10 \mathrm{mM}$; pH 7.4), aerated with $95 \% \mathrm{O}_{2}$ and $5 \% \mathrm{CO}_{2}$. $\mathrm{K}-\mathrm{H}$ ssolution temperature was kept at $37^{\circ} \mathrm{C}$ in order to avoid temperature effect on RR intervals. The isolated heart itself was submerged in $\mathrm{K}-\mathrm{H}$ solution in bath tempered at $37^{\circ} \mathrm{C}$.

Twenty minutes long stabilization period (STAB) 
preceded electrogram recording to avoid the effect of heart preparation. Immediately after STAB, experimental protocol continued with two 40-minutes-lasting consecutive phases: haloperidol exposure (HALO) and washout (WASH) with K-H solution. Haloperidol diluted in $\mathrm{K}-\mathrm{H}$ solution was administered at the concentration of $10 \mathrm{nmol} / \mathrm{L}$.

\subsection{Electrogram acquisition}

Electrograms (EG) were recorded by touch-less method [5] with argent-chloride electrodes (3 orthogonal EG channels) placed in the inner surface of bath and immersed in conductive solution. Electrograms were continually recorded with sampling frequency of $5 \mathrm{kHz}$ and 12-bit resolution by an acquisition card (NI USB-6229 BNC, National Instruments, TX, USA). A custom-build software (sketch in LabView 2010) provided reliable electrogram acquisition.

\subsection{R-peaks detection, tachograms}

Electrogram processing and analysis was performed offline in MATLAB R2015b (MathWorks, MA, USA). The positions of R-peaks were detected by QRS SEEKER [6] detector (Matlab R2013a, MathWorks) utilizing automatic detection method based on wavelet transformation. Detection was carefully reviewed by human specialist.

Series of ten-minutes-long tachograms were obtained from RR series for each experiment, as follows: 1 x 10 minutes of stabilization (STAB), 4 x 10 minutes of haloperidol exposure (HALO), 4 x 10 minutes of washout (WASH). Trends in tachograms were removed by smoothness prior method before further processing.

\subsection{HRV parameters}

HRV parameters recommended by Task Force society [7] were computed by HRVAS software [8].

A number of time domain, frequency domain and nonlinear parameters were analysed, namely: mean, median and standard deviation of RR interval, root mean square of successive differences of RR interval, number of successive RR intervals differing more than $20 \mathrm{~ms}$ (NN20), baseline width of the RR histogram evaluated through triangular interpolation, powers and peaks of very low frequency $(0-0.04 \mathrm{~Hz})$, low frequency $(0.04-0.15 \mathrm{~Hz})$, high frequency $(0.15-0.4 \mathrm{~Hz})$, standard deviations of the points perpendicular to the line-of-identity and along the line-of-identity of Poincaré diagram, sample entropy of RR series and short-term and long-term fluctuation of RR series computed by detrended fluctuation analysis.

\section{Results}

A total of $95 \mathrm{HRV}$ parameters in time, frequency, geometric, and non-linear domain were computed. Only the most informative results are further depicted in paper.

The main finding is that RR intervals remain roughly the same in SCH group after haloperidol administration. Contrary, the RR intervals were prolonged ( $9 \%$ increment in mean, $8 \%$ increment in median) in CON group, as shown in Figure 1. Prolongation of RR interval is gradual and takes about twenty minutes to stabilize at a constant value. The washout did not cause any significant changes in the duration of the RR intervals in both $\mathrm{SCH}$ and CON groups. This indicates that administration of haloperidol irreversible changes RR interval in the CON group.
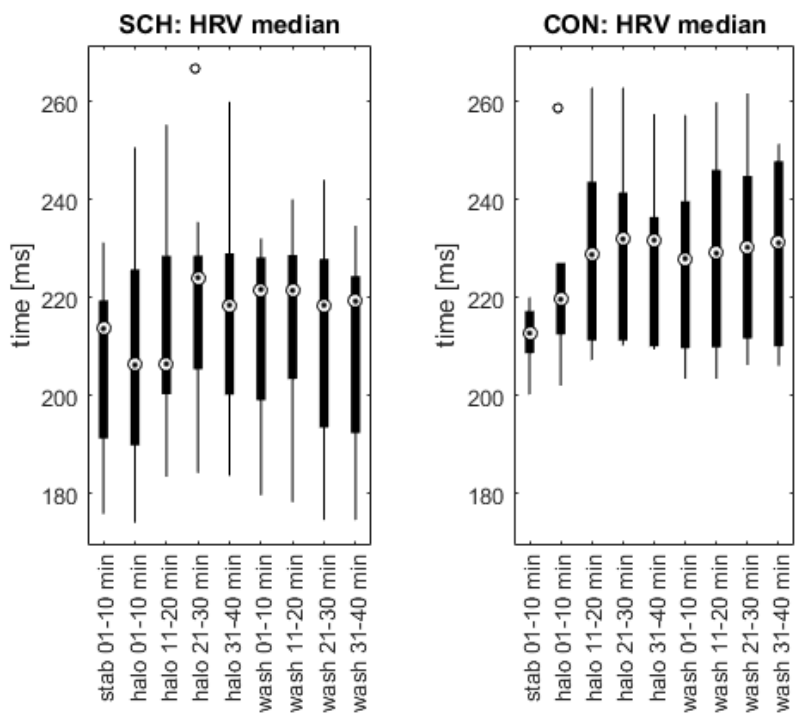

Figure 1. Boxplots of HRV median for schizophrenia-like ( $\mathrm{SCH}$, left) rat isolated hearts and control hearts $(\mathrm{CON}$, right) in stabilization (STAB), haloperidol exposition (HALO) and washout (WASH) periods.

Haloperidol administration in SCH group slightly decreased HRV, expressed as SDNN. The SDNN gradually declined during whole period of haloperidol exposition, as shown in Figure 2.

The same trend, but with stronger effect, has been observed in CON group - haloperidol administration significantly reduced SDNN in CON group (48\% reduction), indicating that heart becomes to beat more regularly. The SDNN was further exponentially decreased in CON group during the whole period of haloperidol exposition until washout.

The moment of washout significantly increased SDNN in both $\mathrm{SCH}$ and $\mathrm{CON}$ group, but SDNN values did not reach initial values in stabilization period and they felt down again during continuing washout in both groups. 

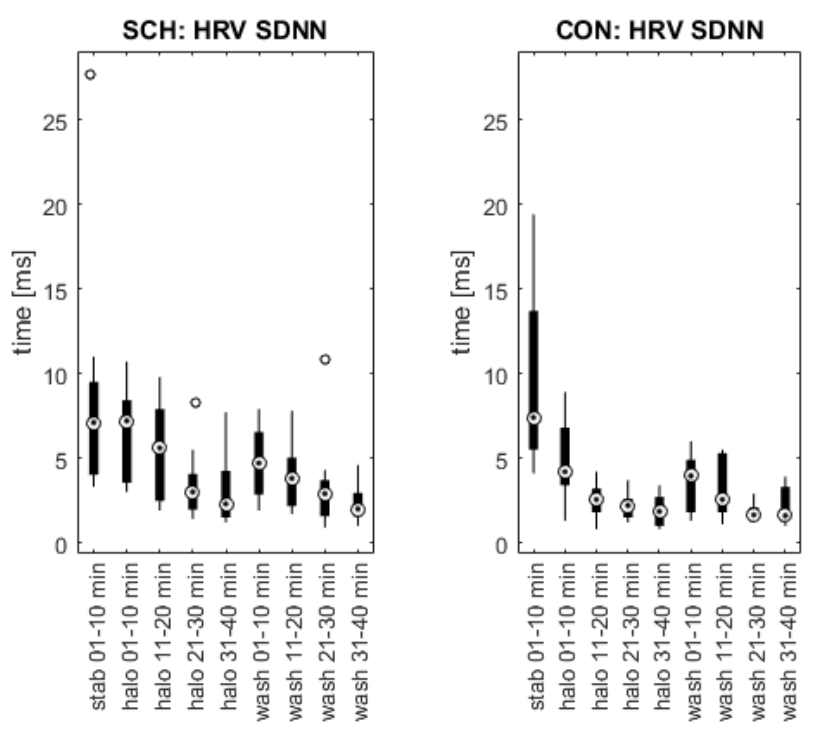

Figure 2. Boxplots of SDNN for schizophrenia-like (SCH, left) rat isolated hearts and control hearts (CON, right) in stabilization (STAB), haloperidol exposition (HALO) and washout (WASH) periods.
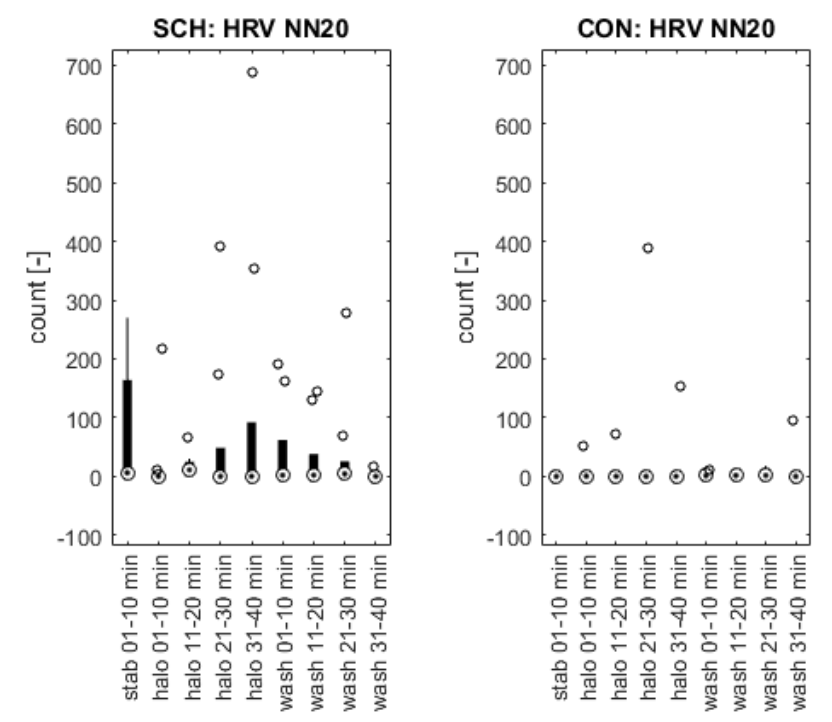

Figure 3. Boxplots of NN20 for schizophrenia-like (SCH, left) rat isolated hearts and control hearts $(\mathrm{CON}$, right) in stabilization (STAB), haloperidol exposition (HALO) and washout (WASH) periods.

Haloperidol administration in SCH group suppressed occasional occurrence of prolonged RR interval. The NN20 parameter, representing the number of RR intervals $20 \mathrm{~ms}$ longer than mean RR interval, was rapidly reduced (97\% reduction) immediately after haloperidol administration in $\mathrm{SCH}$ group, as shown in Figure 3.
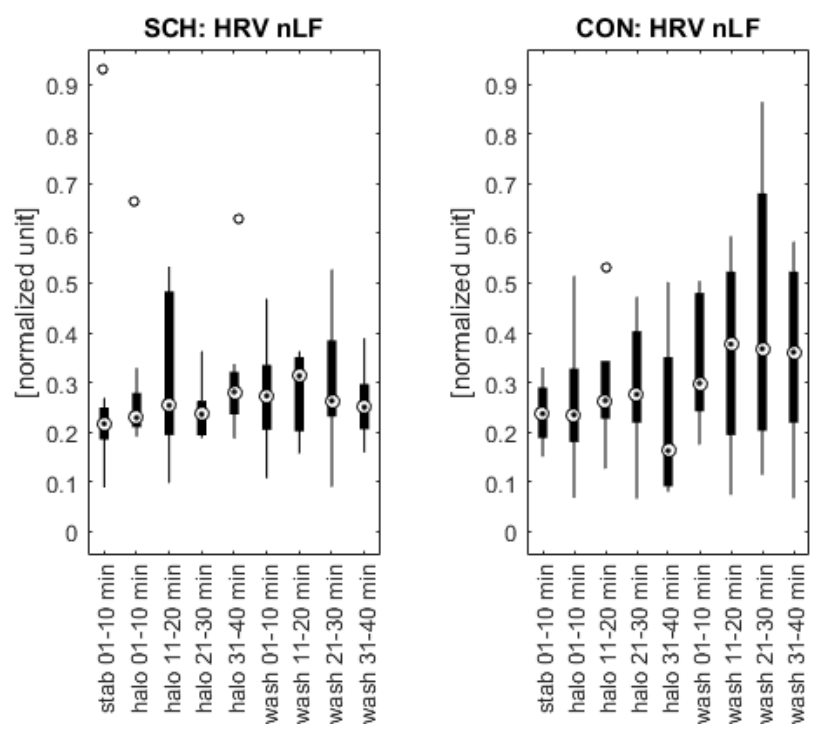

Figure 4. Boxplots of nLF for schizophrenia-like $(\mathrm{SCH}$, left) rat isolated hearts and control hearts ( $\mathrm{CON}$, right) in stabilization (STAB), haloperidol exposition (HALO) and washout (WASH) periods.
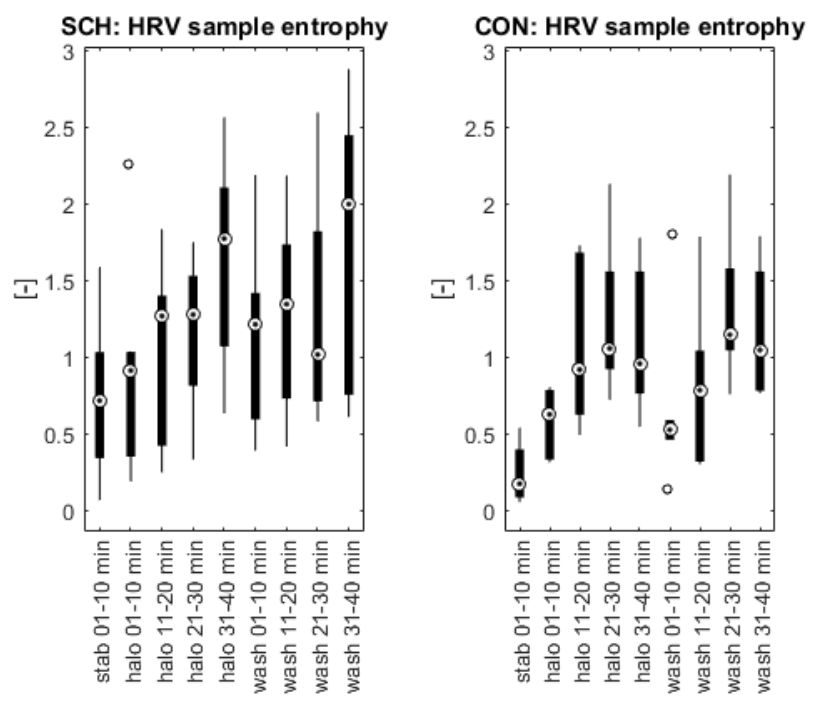

Figure 5. Boxplots of sample entropy for schizophrenialike ( $\mathrm{SCH}$, left) rat isolated hearts and control hearts (CON, right) in stabilization (STAB), haloperidol exposition (HALO) and washout (WASH) periods.

However, the value gradually rise up during the whole HALO period. Washout gradually reduced NN20 in SCH group during the whole washout period.

Haloperidol administration forces heart to beat regularly and avoids rapid changes in RR interval duration in CON group. There is almost no extraordinary beat longer than $20 \mathrm{~ms}$ in CON group during stabilization, haloperidol exposition and washout period. 
Conversely in the CON group, washout incremented low frequency content of tachogram, expressed as normalized LF power (nLF). Those changes were irreversible and persisted even during washout.

Haloperidol administration had only a minor effect on spectral properties of tachogram in the $\mathrm{SCH}$ group, as shown in Figure 4.

Tachogram sample entropy linearly increased during haloperidol administration in both groups, $\mathrm{SCH}$ and $\mathrm{CON}$, as shown in Figure 5. The entropy decreased in both $\mathrm{SCH}$ and CON group immediately at the beginning of the washout, but after that, sample entropy linearly increased during WASH period.

\section{Discussion and conclusion}

Cardiac autonomic dysregulation and reduced complexity of HRV have been found in patients with schizophrenia [9]. It is not known yet, however, whether autonomic dysregulation is the result of schizophrenia, or vice versa. Moreover, not only autonomic nervous system can reduce HRV, but also intrinsic mechanisms of the heart itself. The first finding in this study is that schizophrenialike isolated hearts have higher complexity of HRV than control ones. This finding is in contrast to observed reduced complexity of HRV in in-vivo human heart [8], indicating that autonomous nervous system probably plays a dominant role in HRV reduction of schizophrenia patients, not the intrinsic mechanisms of the heart.

Antipsychotic drug haloperidol used for schizophrenia treatment rapidly suppressed HRV in schizophrenia-like hearts, indicating that haloperidol forces the heart to beat very regularly. There is no evidence in our results, that haloperidol has an arrhythmogenic effect on schizophrenia-like hearts or that it affects RR interval duration. There is also only a minor effect of haloperidol administration on HRV power spectra distribution in schizophrenia-like hearts.

However, haloperidol administration significantly affects HRV in control rat hearts. Haloperidol prolongs RR intervals and induces irregularities in RR intervals, manifesting in higher complexity of HRV.

The effect of haloperidol administration is irreversible both in schizophrenia-like and control hearts, since the washout does not affect HRV parameters in both groups.

It should be emphasized that all abovementioned results apply to isolated heart model only, in which the sympathovagal modulation is completely absent in contrast to invivo situation.

\section{Acknowledgements}

This study was supported by the projects "Kardiovaskulární systém očima molekulární fyziologie" no. MUNI/A/1355/2016 and "Experimentální a translační farmakologický výzkum a vývoj” no. MUNI/A/1063/2016 of the Specific University Research Grant, "Analysis of Relationship between Electrical Activity and Blood Flow at the Heart Ventricles" number GAP102/12/2034, and "Aplikace pokročilých prístupů pro analýzu signálů, systémů a dat v lékařských, biologických a bioinformatických oblastech" no. FEKT-S-17-4487.

\section{References}

[1] Stracina et al., Long-Term Haloperidol Treatment Prolongs QT Interval and Increases Expression of Sigma 1 and IP3 Receptors in Guinea Pig Hearts, Tohoku J Exp Med. 2015;236(3):199-207

[2] Wu CS, Tsai YT, Tsai HJ. Antipsychotic drugs and the risk of ventricular arrhythmia and/or sudden cardiac death: a nation-wide case-crossover study. J Am Heart Assoc 2015;4:e001568.

[3] Fujii K, Ozeki Y, Okayasu H, Takano Y, Shinozaki T, Hori H, Orui M, Horie M, Kunugi H, Shimoda K. QT is longer in drug-free patients with schizophrenia compared with age-matched healthy subjects. PLoS One 2014;9(6):e98555.

[4] Lodge DJ, Grace AA. Gestational methylazoxymethanol acetate administration: a developmental disruption model of schizophrenia. Behav Brain Res 2009;204:306-12.

[5] Kolářová J, Fialová K, Janoušek O, Nováková M, Provazník I. Experimental methods for simultaneous measurement of action potentials and electrograms in isolated heart. Physiol Res 2010;59(S1):71-80

[6] Vítek M, Kozumplík J: QRS seeker, Brno University of Technology.

[7] Task Force of The European Society of Cardiology and The North American Society of Pacing and Electrophysiology. Heart Rate Variability, Standards of measurement, physiological interpretation and clinical use. Europ Heart Journal 1996;354-381.

[8] Ramshur, JT. Design, Evaluation, and Application of Heart Rate Variability Software (HRVAS). The University of Memphis, 2010.

[9] Montaquila JM, Trachik BJ, Bedwell JS. Heart rate variability and vagal tone in schizophrenia: A review. Journal of Psychiatric Research 2015,69:57-66.

Address for correspondence.

Oto Janousek

Department of Biomedical Engineering

The Faculty of Electrical Engineering and Communication

Brno University of Technology

Technicka 3082/12

61600 Brno

Czech Republic

janouseko@feec.vutbr.cz 